\title{
ASTROPHYSICAL TESTS OF LORENTZ AND CPT VIOLATION WITH PHOTONS
}

\author{
V. Alan Kosteleckí ${ }^{1}$ and Matthew Mewes ${ }^{2}$ \\ Received 2008 September 17; accepted 2008 October 21; published 2008 November 13
}

\begin{abstract}
A general framework for tests of Lorentz invariance with electromagnetic waves is presented, allowing for operators of arbitrary mass dimension. Signatures of Lorentz violations include vacuum birefringence, vacuum dispersion, and anisotropies. Sensitive searches for violations using sources such as active galaxies, gamma-ray bursts, and the cosmic microwave background are discussed. Direction-dependent dispersion constraints are obtained on operators of dimension 6 and 8 using gamma-ray bursts and the blazar Markarian 501. Stringent constraints on operators of dimension 3 are found using 5 year data from the Wilkinson Microwave Anisotropy Probe. No evidence appears for isotropic Lorentz violation, while some support at $1 \sigma$ is found for anisotropic violation.
\end{abstract}

Subject headings: cosmic microwave background — galaxies: active — gamma rays: bursts — gravitation — relativity

Recent years have seen a resurgence in tests of relativity, spurred in part by the prospect of relativity violations arising in a unified description of nature (Kostelecký \& Samuel 1989; Kostelecký \& Potting 1991). Experimental searches for violations of Lorentz invariance, the symmetry underlying relativity, have been performed in a wide range of systems (for data tables see Kostelecký \& Russell 2008). Historically, experiments probing the behavior of light have been central in confirming relativity. Contemporary versions of the classic Michelson-Morley and Kennedy-Thorndike experiments (Lipa et al. 2003; Antonini et al. 2005; Müller et al. 2007) remain among the most sensitive tests today.

Some tight constraints on relativity violations have been achieved by seeking tiny changes in light that has propagated over astrophysical distances. Many of these search for a change in polarization resulting from vacuum birefringence, using sources such as galaxies (Carroll et al. 1990; Colladay \& Kostelecký 1998; Kostelecký \& Mewes 2001, 2002), gamma-ray bursts (GRBs; Mitrofanov 2003; Jacobson et al. 2004; Kostelecký \& Mewes 2006; Kahniashvili et al. 2006; Fan et al. 2007), and the cosmic microwave background (CMB; Feng et al. 2006; Gamboa et al. 2006; Kostelecký \& Mewes 2007; Cabella et al. 2007; Komatsu et al. 2009; Xia et al. 2008; Kahniashvili et al. 2008). Others seek a frequency-dependent velocity arising from vacuum dispersion, using GRBs, pulsars, and blazars (Amelino-Camelia et al. 1998; Kostelecký \& Mewes 2002; Boggs et al. 2004; Martínez \& Piran 2006; Ellis et al. 2006; Lamon et al. 2008; Albert et al. 2008). Here, we present a general theoretical framework that characterizes Lorentz-violating effects on the vacuum propagation of electromagnetic waves and includes operators of all mass dimensions. We discuss several techniques that can be used to search for the unconventional signals of Lorentz violation, Using vacuumdispersion constraints from GRBs and the blazar Markarian 501, we place new direction-dependent limits on several combinations of coefficients for Lorentz violation. We also perform a search for Lorentz violations in the 5 year results from the Wilkinson Microwave Anisotropy Probe (WMAP; Komatsu et al. 2009; Hinshaw et al. 2009; Nolta et al. 2009), finding some

\footnotetext{
${ }^{1}$ Physics Department, Indiana University, Bloomington, IN 47405.

${ }^{2}$ Physics Department, Marquette University, Milwaukee, WI 53201.
}

evidence for anisotropic violations but no support for isotropic violations.

At attainable energies, violations of Lorentz invariance are described by a framework called the standard-model extension (SME; Colladay \& Kostelecký 1997, 1998; Kostelecký 2004) that is based on effective field theory (Kostelecký \& Potting 1995). Approaches outside field theory also exist (AmelinoCamelia 2008). The SME characterizes all realistic violations affecting known particles and fields, while incorporating otherwise established physics. Much of the work on Lorentz violation has focused on the minimal SME, which restricts attention to gauge-invariant operators of renormalizable dimension. In this work, we consider the gauge-invariant purephoton sector of the full SME with Lorentz-violating operators of arbitrary dimension, which has Lagrange density (Kostelecký \& Mewes 2007)

$$
\mathcal{L}=-\frac{1}{4} F_{\mu \nu} F^{\mu \nu}+\frac{1}{2} \epsilon^{\kappa \lambda \mu \nu} A_{\lambda}\left(\hat{\boldsymbol{k}}_{A F}\right)_{\kappa} F_{\mu \nu}-\frac{1}{4} F_{\kappa \lambda}\left(\hat{\boldsymbol{k}}_{F}\right)^{\kappa \lambda \mu \nu} F_{\mu \nu},
$$

where $A_{\mu}$ is the 4-potential with field strength $F_{\mu \nu}$. In a flat background with energy-momentum conservation, the Lorentz violation arises through the differential operators

$$
\begin{aligned}
& \left(\hat{\boldsymbol{k}}_{A F}\right)_{\kappa}=\sum_{d=\mathrm{odd}}\left(k_{A F}^{(d)}\right)_{\kappa}^{\alpha_{1} \ldots \alpha_{(d-3)}} \partial_{\alpha_{1}} \ldots \partial_{\alpha_{(d-3)}}, \\
& \left(\hat{\boldsymbol{k}}_{F}\right)^{\kappa \lambda \mu \nu}=\sum_{d=\text { even }}\left(k_{F}^{(d)}\right)^{\kappa \lambda \mu \nu \alpha_{1} \ldots \alpha_{(d-4)}} \partial_{\alpha_{1}} \ldots \partial_{\alpha_{(d-4)}} .
\end{aligned}
$$

The constant coefficients $\left(k_{A F}^{(d)}\right)^{\alpha_{1} \ldots \alpha_{(d-3)}}$ and $\left(k_{F}^{(d)}\right)^{\kappa \lambda \mu \nu \alpha_{1} \ldots \alpha_{(d-4)}}$ characterize the degree of Lorentz violation. The former control CPT-odd operators and are nonzero for odd dimension $d \geq 3$, while the latter control CPT-even operators and are restricted to even $d \geq 4$.

The Lagrange density (eq. [1]) yields modified Maxwell equations. At leading order in coefficients for Lorentz violation, two plane-wave solutions exist. The corresponding two modified dispersion relations can be written in the form

$$
p(\omega) \approx\left[1+s^{0} \mp \sqrt{\left(s^{1}\right)^{2}+\left(s^{2}\right)^{2}+\left(s^{3}\right)^{2}}\right] \omega,
$$


where $p$ and $\omega$ are the wavenumber and frequency, respectively. It follows that electromagnetic waves generically contain two propagating modes with different velocities and polarizations. The symbols $s^{0}, s^{1}, s^{2}$, and $s^{3}$ represent certain combinations of coefficients for Lorentz violation, and they depend on the frequency $\omega$ and direction of propagation $\hat{\boldsymbol{p}}$. With convenient normalizations, $s^{1}, s^{2}$, and $s^{3}$ are the Stokes parameters $s^{1}=$ $Q, s^{2}=U$, and $s^{3}=V$ of the faster mode, while $s^{0}$ is a scalar combination analogous to the intensity $s^{0}=I$. These four combinations completely control the leading-order effects of Lorentz violation on light propagating through empty space. The combination $s^{3}$ depends only on the coefficients $\left(k_{A F}^{(d)}\right)^{\alpha_{1} \ldots \alpha_{(d-3)}}$, while $\varsigma^{0}, s^{1}$, and $\varsigma^{2}$ depend only on the coefficients $\left(k_{F}^{(d)}\right)^{\kappa \lambda \mu \nu \alpha_{1} \ldots \alpha_{(d-4)}}$.

It is convenient to identify a minimal set of coefficient combinations that affect light propagating in vacuo. This can be accomplished through spherical-harmonic decomposition. Since $s^{0}$ and $s^{3}$ are rotation scalars while $s^{1}$ and $s^{2}$ are rotation tensors, their decomposition must involve some form of tensor spherical harmonics. The spin-weighted harmonics ${ }_{s} Y_{j m}(\hat{\boldsymbol{p}})$ provide a well-understood set (Newman \& Penrose 1966; Goldberg 1967). The index $s$ is the spin weight, which up to a sign is equivalent to helicity. Decomposing yields

$$
\begin{aligned}
\boldsymbol{\varsigma}^{0} & =\sum_{d j m} \omega^{d-4}{ }_{0} Y_{j m}(\hat{\boldsymbol{n}}) k_{(I) j m}^{(d)}, \\
\boldsymbol{\varsigma}^{1} \pm i \boldsymbol{\varsigma}^{2} & =\sum_{d j m} \omega^{d-4}{ }_{ \pm 2} Y_{j m}(\hat{\boldsymbol{n}})\left(k_{(E) j m}^{(d)} \mp i k_{(B) j m}^{(d)}\right), \\
\boldsymbol{\varsigma}^{3} & =\sum_{d j m} \omega^{d-4}{ }_{0} Y_{j m}(\hat{\boldsymbol{n}}) k_{(V) j m}^{(d)},
\end{aligned}
$$

where $j \leq d-2$ and $\hat{\boldsymbol{n}}=-\hat{\boldsymbol{p}}$ is a unit vector pointing to the source in astrophysics tests.

With this decomposition, all types of Lorentz violations for propagation in vacuo can now be simply characterized using four sets of spherical coefficients, $k_{(I) j m}^{(d)}, k_{(E) j m}^{(d)}$, and $k_{(B) j m}^{(d)}$ for CPT-even effects and $k_{(V) j m}^{(d)}$ for CPT-odd effects. For each coefficient, the underlying Lorentz-violating operator has mass dimension $d$ and eigenvalues of total angular momentum given by jm, as usual. For light from astrophysical sources, dispersion arises when the speed of propagation depends on frequency, which occurs for any nonzero coefficient with $d \neq 4$. Birefringence results when the usual degeneracy among polarizations is broken, for which at least one of $k_{(E) j m}^{(d)}, k_{(B) j m}^{(d)}$, or $k_{(V) j m}^{(d)}$ is nonzero. For example, all operators producing lightspeed corrections that are linear in the energy have $d=5$ and are necessarily birefringent. The only coefficients for nonbirefringent dispersion are therefore $k_{(I) j m}^{(d)}$ with even $d \geq 6$. Since birefringence tests using polarimetry are typically many orders of magnitude more sensitive than dispersion tests using timing, in the following discussion of dispersion we focus only on coefficients for nonbirefringent dispersion.

Tests for vacuum dispersion seek differences in the velocity of light at different wavelengths. In the present context with zero birefringent coefficients, the change in velocity is $\delta v \simeq$ $-\varsigma^{0}$. We see from equation (5) that the velocity generically depends on the direction $\hat{\boldsymbol{n}}$ as well as the frequency $\omega$. Typical analyses study explosive or pulsed sources of radiation producing light over a wide wavelength range in short time periods, comparing the arrival times of different wavelengths. This idea has been the focus of many searches based on modified dispersion relations (Amelino-Camelia et al. 1998; Kostelecký \& Mewes 2002; Boggs et al. 2004; Martínez \& Piran 2006; Ellis et al. 2006; Lamon et al. 2008; Albert et al. 2008). Many of these studies assume isotropic violations, which corresponds to the limit $j=m=0$. However, at each dimension $d$, this isotropic restriction misses $\left(d^{2}-2 d-2\right)$ possible effects from anisotropic violations.

To calculate arrival-time differences in an expanding universe, some care is required (Jacob \& Piran 2008). In the present case, the photons propagate between two comoving objects, so the relevant coordinate interval is $d l_{c}=(1+z) d l_{p}=-v_{z} d z / H_{z}$. Here, $v_{z}$ is the particle velocity at redshift $z$, and $H_{z}=H_{0}\left(\Omega_{r} \zeta^{4}+\right.$ $\left.\Omega_{m} \zeta^{3}+\Omega_{k} \zeta^{2}+\Omega_{\Lambda}\right)^{1 / 2}$ with $\zeta=1+z$ is the Hubble expansion rate at $z$ in terms of the present-day Hubble constant $H_{0} \simeq$ $71 \mathrm{~km} \mathrm{~s}^{-1} \mathrm{Mpc}^{-1}$, radiation density $\Omega_{r} \simeq 0$, matter density $\Omega_{m} \simeq 0.27$, vacuum density $\Omega_{\Lambda} \simeq 0.73$, and curvature density $\Omega_{k}=1-\Omega_{r}-\Omega_{m}-\Omega_{\Lambda}$. The total coordinate distance is the same for all wavelengths, but the travel times may differ. Integrating $d l_{c}$ from the same initial time to the two arrival times for the two velocities gives a relation for the arrival-time difference $\Delta t$, which depends on the two energies and the source location on the sky. For the present case with Lorentz violation at dimension $d$, we find

$$
\Delta t \approx-\Delta \omega^{d-4} \int_{0}^{z} \frac{(1+z)^{d-4}}{H_{z}} d z \sum_{j m}{ }_{0} Y_{j m}(\hat{\boldsymbol{n}}) k_{(I) j m}^{(d)},
$$

where $\Delta \omega^{d-4}$ is the difference in $\omega^{d-4}$ between the two frequencies.

As an illustration, consider the bright gamma-ray burst GRB 021206 at right ascension $240^{\circ}$ and declination $-9.7^{\circ}$. Over energies from 3 to $17 \mathrm{MeV}$, arrival-time differences are no more than $\Delta t<4.8 \mathrm{~ms}$ for this source at $z \simeq 0.3$ (Boggs et al. 2004). Numerical integration of equation (6) leads to a bound on one direction-specific combination of the 25 independent coefficients for nonbirefringent dispersion with $d=6$,

$$
\sum_{j m}{ }_{0} Y_{j m}\left(99.7^{\circ}, 240^{\circ}\right) k_{(I) j m}^{(6)}<1 \times 10^{-16} \mathrm{GeV}^{-2} .
$$

For the 63 independent nonbirefringent dispersive operators with $d=8$, we obtain

$$
\sum_{j m}{ }_{0} Y_{j m}\left(99.7^{\circ}, 240^{\circ}\right) k_{(I) j m}^{(8)}<3 \times 10^{-13} \mathrm{GeV}^{-4}
$$

Operators with higher $d$ can be treated similarly. Note that many sources are required to constrain fully the coefficient space for a given $d$. In contrast, only one source is needed to constrain fully the corresponding coefficient in the restrictive isotropic limit $j=m=0$. In this limit, the bounds from equations (7) and (8) reduce to $k_{(I) 00}^{(6)}<4 \times 10^{-16} \mathrm{GeV}^{-2}$ and $k_{(I) 00}^{(8)}<9 \times$ $10^{-13} \mathrm{GeV}^{-4}$, respectively.

As another example, consider Markarian 501, which lies at $z \simeq 0.03$. This source produces flares with photon energies in the $\mathrm{TeV}$ range, making it particularly sensitive to an energydependent velocity and also to threshold analyses (AmelinoCamelia \& Piran 2001). A recent analysis of observations by the MAGIC collaboration found some evidence for a nonbirefringent velocity defect of the form $\delta v=-\omega / M$ or $\delta v=$ $-\omega^{2} / M^{2}$ (Albert et al. 2008). The first case is incompatible with the present treatment; a reanalysis incorporating the necessary birefringence could yield comparatively weak but compatible new bounds. The second case suggests dispersion with $M \simeq$ $\left(6_{-1}^{+5}\right) \times 10^{10} \mathrm{GeV}$, assuming an arrival-time lag due entirely to 
nonbirefringent Lorentz violation. For $d=6$, this yields the single constraint

$$
\sum_{j m}{ }_{0} Y_{j m}\left(50.2^{\circ}, 253^{\circ}\right) k_{(I) j m}^{(6)} \simeq\left(3_{-2}^{+1}\right) \times 10^{-22} \mathrm{GeV}^{-2},
$$

consistent with the GRB bound (eq. [7]). In the isotropic limit, this becomes $k_{(I) 00}^{(6)} \simeq\left(10_{-7}^{+4}\right) \times 10^{-22} \mathrm{GeV}^{-2}$.

Next, we consider tests for vacuum birefringence. In birefringent scenarios, the two plane-wave eigenmodes travel at slightly different velocities, which alters their superposition and hence the net polarization of the light as it propagates in free space. The polarization change is equivalent to a rotation of the Stokes vector $s=\left(s^{1}, s^{2}, s^{3}\right)^{T}$ about the birefringent axis $\mathbf{s}=\left(\mathrm{s}^{1}, \mathrm{~s}^{2}, \mathrm{~s}^{3}\right)^{T}$. The total rotation angle is equal to the relative phase change between the two eigenmodes. Infinitesimally, the rate of rotation is $d \boldsymbol{s} / d t=-i \boldsymbol{\Sigma} \cdot \boldsymbol{s}$, where $\boldsymbol{\Sigma}^{a b}=-2 i \omega \epsilon^{a b c} \boldsymbol{s}^{c}$ is the rotation generator. Integration from source redshift $z$ to 0 taking into account the cosmological expansion yields the net change in the Stokes vector,

$$
\Delta \boldsymbol{s}=\int_{z}^{0} \frac{i \boldsymbol{\Sigma}_{z} \cdot \boldsymbol{s}}{(1+z) H_{z}} d z,
$$

where $\boldsymbol{\Sigma}_{z}$ is the rotation matrix at the blueshifted frequency $(1+z) \omega$ and source direction $\hat{\boldsymbol{n}}$. The net polarization change $\Delta s$ can depend on frequency and direction of propagation. To seek birefringence, we can either model the polarization at the source and seek discrepancies in the observed polarization, or we can test for unexpected frequency dependences.

In what follows, we investigate vacuum birefringence via the CMB, leaving the use of GRB polarimetry in this context to be discussed elsewhere (V. A. Kostelecký \& M. Mewes in preparation). The CMB has a long baseline but comparatively low frequency, which implies lesser sensitivities to $d>3$ violations relative to higher frequency sources. Here, we focus on the four $d=3$ Lorentz-violating operators. These induce energy-independent polarization changes, so the best constraints are expected from the most distant sources irrespective of frequency. The CMB therefore has the potential to yield maximal sensitivity to these CPT-odd operators. For any CPTodd case, birefringence causes a rotation of the Stokes vector about the $s^{3}$ axis, corresponding to a rotation of the linearpolarization angle $\psi$ with no change in the degree of linear or circular polarization. For $d=3$, the value of $\psi$ at present is $\psi=\psi_{z}+\delta \psi_{z}$, where $\psi_{z}$ is the blueshifted angle and $\delta \psi_{z}$ is its rotation,

$$
\delta \psi_{z}=\int_{0}^{z} \frac{d z}{(1+z) H_{z}} \sum_{j m}{ }_{0} Y_{j m}(\hat{\boldsymbol{n}}) k_{(V) j m}^{(3)}
$$

Taking $z=1100$ for the CMB and including a small radiation component $\Omega_{r} \simeq 0.015$, the rotation reduces to the directiondependent approximation

$$
\delta \psi_{\mathrm{CMB}} \simeq 3.5^{\circ} \times 10^{43} \mathrm{GeV}^{-1} \sum_{j m}{ }_{0} Y_{j m}(\hat{\boldsymbol{n}}) k_{(V) j m}^{(3)} .
$$

We remark in passing that CPT-even operators produce a complicated mixing of circular and linear polarization, rather than a simple rotation of $\psi$ (V. A. Kostelecký \& M. Mewes in preparation).

We next search for the above effect in the recent WMAP 5 year results (Hinshaw et al. 2009; Nolta et al. 2009). We gen- erate initial sky maps of the Stokes parameters using the bestfit correlation coefficients $C_{j}$ as calculated by the WMAP collaboration within the $\Lambda \mathrm{CDM}$ model assuming Gaussianality. The Stokes parameters at each point on the sky are then rotated appropriately and used to calculate the $C_{j}$ coefficients at the present epoch. The likelihood of these coefficients is determined using available WMAP software. The underlying cosmology is kept fixed, so we are comparing the likelihood of Lorentz violation relative to a reasonable Lorentz-invariant cosmology. Our analysis uses TE and TB data at high- $l$ corresponding to $j=24-450$, disregarding the $T T$ data. The latter is a good approximation, because the $T T$ data would dominate an analysis with varying cosmology and therefore hold the cosmology comparatively fixed.

The correlation coefficients $C_{j}$ are rotationally invariant, so our analysis has sensitivity only to rotationally invariant combinations of Lorentz-violating coefficients. In the present context, these are the isotropic coefficient $\left(k_{A F}^{(3)}\right)^{T}=-k_{(V) 00}^{(3)} / \sqrt{4 \pi}$ and the scalar magnitude $\left|\boldsymbol{k}_{A F}^{(3)}\right|=\left(6\left|k_{(V) 11}^{(3)}\right|^{2}+\right.$ $\left.3\left|k_{(V) 10}^{(3)}\right|^{2}\right)^{1 / 2} / \sqrt{4 \pi}$. In particular, our results are independent of the direction of $\boldsymbol{k}_{\boldsymbol{A} \boldsymbol{F}}^{(3)}$. Although the analysis contains no a priori anisotropies, the procedure involves generating random realizations that contain anisotropies. As a result, the likelihood $L\left(k_{(V) j m}^{(3)}, r\right)$ for a given realization $r$ is anisotropic. In obtaining the total likelihood for a given set of coefficients for Lorentz violation, we sum over the likelihoods of all possible realizations weighted by the probability density $P(r)$, yielding $L\left(k_{(V) j m}^{(3)}\right)=\sum_{r} P(r) L\left(k_{(V) j m}^{(3)}, r\right)$. This total likelihood is simply the average over all possible universes and is a rotationally invariant indicator of Lorentz violation. Here, we estimate $L\left(k_{(V) j m)}^{(3)}\right)$ for a range of values of $k_{(V) j m}^{(3)}$ by averaging over 3000 realizations per value. The results for the four coefficients with $d=3$ are shown in Figure 1.

For the isotropic coefficient, we obtain the $1 \sigma$ result

$$
k_{(V) 00}^{(3)}=(2.3 \pm 5.4) \times 10^{-43} \mathrm{GeV} .
$$

This improves by about an order of magnitude on the previous limit from radio galaxy data (Carroll et al. 1990). The result is also consistent with that of the WMAP collaboration, which found a rotation of $\delta \psi \simeq 1.2^{\circ} \pm 2.2^{\circ}$ (Komatsu et al. 2009) corresponding to $k_{(V) 00}^{(3)}<(1.2 \pm 2.2) \times 10^{-43} \mathrm{GeV}$, and that of another recent analysis yielding $k_{(V) 00}^{(3)}<2.5 \times 10^{-43} \mathrm{GeV}$ (Kahniashvili et al. 2008). Other reported limits obtained from WMAP 3 year data (Page et al. 2007) include $k_{(V) 00}^{(3)}=$ $(6.0 \pm 4.0) \times 10^{-43} \mathrm{GeV}$ (Feng et al. 2006) and $k_{(V) 00}^{(3)}=$ $(2.5 \pm 3.0) \times 10^{-43} \mathrm{GeV}$ (Cabella et al. 2007). Some indication of a nonzero rotation has been found in previous studies. One involving BOOMERANG (hereafter B03; see Montroy et al. 2006; Piacentini et al. 2006) data alone yielded the possibility $k_{(V) 00}^{(3)}=(12 \pm 7) \times 10^{-43} \mathrm{GeV}$ (Kostelecký \& Mewes 2007), while the result from another analysis combining B03 and WMAP 5 year data corresponds to $k_{(V) 00}^{(3)}<(2.6 \pm 1.9) \times$ $10^{-43} \mathrm{GeV}$ (Xia et al. 2008). While consistent with the latter, our result (eq. [13]) shows little evidence for isotropic Lorentz violation.

For each anisotropic coefficient, Figure 1 displays the likelihood. As expected, the results are independent of the direction of $\boldsymbol{k}_{\boldsymbol{A} \boldsymbol{F}}^{(3)}$. The plot symmetries reflect the expected behavior under $\boldsymbol{k}_{\boldsymbol{A} \boldsymbol{F}}^{(3)} \rightarrow-\boldsymbol{k}_{\boldsymbol{A} \boldsymbol{F}}^{(3)}$. These plots yield the result

$$
\left|\boldsymbol{k}_{\boldsymbol{A F}}^{(3)}\right|=\left(10_{-8}^{+4}\right) \times 10^{-43} \mathrm{GeV},
$$

revealing some evidence at $1 \sigma$ for anisotropic Lorentz violation 

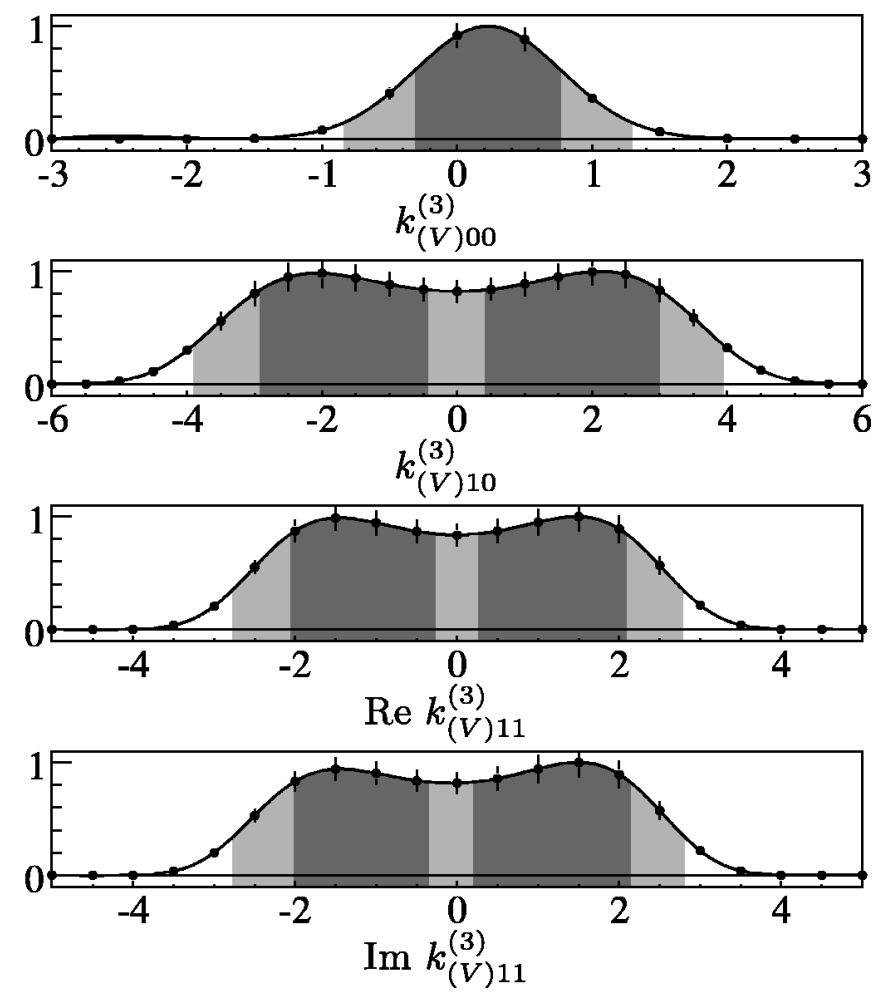

FIG. 1.-Relative likelihood vs. the four $d=3$ coefficients for Lorentz violation. Points indicate the values at which the ensemble averages were made, and the bars represent their standard errors. The line is an extrapolation through the points. Dark-gray regions indicate the estimated 68\% confidence interval, while the light-gray region shows the $95 \%$ level. All coefficients are in units of $10^{-42} \mathrm{GeV}$. in the WMAP 5 year data. This agrees with the indication of anisotropic Lorentz violation found from an analysis of B03 data, which corresponds to $\left|\boldsymbol{k}_{\boldsymbol{A} \boldsymbol{F}}^{(3)}\right|=(15 \pm 6) \times 10^{-43} \mathrm{GeV}$ (Kostelecký \& Mewes 2007). The data are consistent with no Lorentz violation at $2 \sigma$, with a $95 \%$ confidence level of $\left|\boldsymbol{k}_{\boldsymbol{A} \boldsymbol{F}}^{(3)}\right|<2 \times 10^{-42} \mathrm{GeV}$. This fully constrains the vector components of $\boldsymbol{k}_{\boldsymbol{A} F}^{(3)}$, and the results in equations (13) and (14) provide a measurement of all four of the $d=3$ coefficients for Lorentz violation.

This work is supported in part by the US DOE under grant DE-FG02-91ER40661.
Albert, J., et al. 2008, Phys. Lett. B, 668, 253

Amelino-Camelia, G. 2008, preprint (arXiv: 0806.0339)

Amelino-Camelia, G., Ellis, J., Mavromatos, N. E., Nanopoulos, D. V., \& Sarkar, S. 1998, Nature, 393, 763

Amelino-Camelia, G., \& Piran, T. 2001, Phys. Rev. D, 64, 036005

Antonini, P., et al. 2005, Phys. Rev. A, 71, 050101

Boggs, S. E., Wunderer, C. B., Hurley, K., \& Coburn, W. 2004, ApJ, 611, L77

Cabella, P., Natoli, P., \& Silk, J. 2007, Phys. Rev. D, 76, 123014

Carroll, S. M., Field, G. B., \& Jackiw, R. 1990, Phys. Rev. D, 41, 1231

Colladay, D., \& Kostelecký, V. A. 1997, Phys. Rev. D, 55, 6760 . 1998, Phys. Rev. D, 58, 116002

Ellis, J., Mavromatos, N. E., Nanopoulos, D. V., Sakharov, A. S., \& Sarkisyan, E. K. G. 2006, Astropart. Phys., 25, 402

Fan, Y.-Z., Wei, D.-M., \& Xu, D. 2007, MNRAS, 376, 1857

Feng, B., Li, M., Xia, J.-Q., Chen, X., \& Zhang, X. 2006, Phys. Rev. Lett., 96, 221302

Gamboa, J., López-Sarrión, J., \& Polychronakos, A. P. 2006, Phys. Lett. B, 634,471

Goldberg, J. N. 1967, J. Math. Phys., 8, 2155

Hinshaw, G., et al. 2009, ApJS, in press (arXiv: 0803.0732)

Jacob, U., \& Piran, T. 2008, J. Cosmol. Astropart. Phys., 1, 31

Jacobson, T., Liberati, S., Mattingly, D., \& Stecker, F. W. 2004, Phys. Rev. Lett., 93, 021101

\section{REFERENCES}

Kahniashvili, T., Durrer, R., \& Maravin, Y. 2008, preprint (arXiv: 0807.2593) Kahniashvili, T. Gogoberidze, G., \& Ratra, B. 2006, Phys. Lett. B, 643, 81

Komatsu, E., et al. 2009, ApJS, in press (arXiv: 0803.0547)

Kostelecký, V. A. 2004, Phys. Rev. D, 69, 105009

Kostelecký, V. A., \& Mewes, M. 2001, Phys. Rev. Lett., 87, 251304

_. 2002, Phys. Rev. D, 66, 056005

- 2006, Phys. Rev. Lett., 97, 140401

- 2007, Phys. Rev. Lett., 99, 011601

Kostelecký, V. A., \& Potting, R. 1991, Nucl. Phys. B, 359, 545 -. 1995, Phys. Rev. D, 51, 3923

Kostelecký, V. A., \& Russell, N. 2008, preprint (arXiv: 0801.0287)

Kostelecký, V. A., \& Samuel, S. 1989, Phys. Rev. D, 39, 683

Lamon, R., Produit, N., \& Steiner, F. 2008, Gen. Relativ. Gravitation, 40, 1731

Lipa, J., et al. 2003, Phys. Rev. Lett., 90, 060403

Martínez, M. R., \& Piran, T. 2006, J. Cosmol. Astropart. Phys., 4, 6

Mitrofanov, I. G. 2003, Nature, 426, 139

Montroy, T. E., et al. 2006, ApJ, 647, 813

Müller, H., et al. 2007, Phys. Rev. Lett., 99, 050401

Newman, E. T. and Penrose, R. 1966, J. Math. Phys., 7, 863

Nolta, M., et al. 2009, ApJS, in press (arXiv: 0803.0593)

Page, L., et al. 2007, ApJS, 170, 335

Piacentini, F., et al. 2006, ApJ, 647, 833

Xia, J.-Q., Li, H., Zhao, G.-B., \& Zhang, X. 2008, ApJ, 679, L61 